\title{
7. Diversity of macromycete communities
}

\author{
Jolanta Adamczyk
}

Forty-six species of macromycetes were identified in the analysed abandoned lands. Communities of macromycetes show a diversity related to the development stage of the communities in the abandoned lands.

The community with lichen and the community with Anthoxanthum aristatum and Corynephorus canescens are the poorest in macrofungi species. There, mostly saprotrophic species of the Bovista, Conocybe, Lycoperdon, Marasmius, $\mathrm{Pa}$ naeolus, and Psilocybe genera occur, individually or in small groups (Tab. 7.1). In study plots with trees, some mycorrhizal species appear, e.g. Amanita muscaria (Photo 7.3), Inocybe corydalina, and Paxillus involutus (Photo 7.1).

Equally poor in macromycete species are the few communities with Calamagrostis epigejos. Sporocarps of saprotrophic species were found there, e.g. Conocybe tenera, Psilocybe semiglobata, and $P$. semilanceata.

In patches of the phytocoenosis with Agrostis capillaris, Hieracium pilosella, and Achillea millefolium, macrofungi form larger and more abundant communities (7-19 species). In study plots where trees grow, there are some mycorrhizal species, which often produce numerous sporocarps. In patches, where the moss layer is present, species associated with mosses were observed, such as Arrhenia lobata, Rickenella fibula (Photo 7.2). In the community with Agrostis capillaris, Hieracium pilosella, and Achillea millefolium, the Glinnik A plot was the most abundant with macrofungi species.

The floristically abundant community with Cirsium arvense, Galium mollugo, and Gnaphalium sylcaticum, features fungi which are richer in macromycete species (10-19 species). It is charac- terised by fairly numerous saprotrophic species, e.g. Clitocybe vibecina, Conocybe tenera, Macrolepiota procera and a large share of mycorrhizal species, e.g. Amanita muscaria, Boletus edulis, Inocybe maculata, Suillus bovinus and Xerocomus badius. Only in this community, the occurrence of fruiting bodies of Hemimycena lactea on pine needles was observed.

Patches of the community with Elymus repens or Poa pratensis, which occurred at study plots $\mathrm{Su}$ lejów A and Sulejów C, feature a quite rich and diverse composition of macrofungi species. Among saprotrophic fungi, species of gasteroid fungi prevail, such as Calvatia excipuliformis, Scleroderma citrina, and Vascellum pratense. Mycorrhizal species also grow here, e.g. Amanita muscaria, Inocybe asterospora, and I. maculata. There are many fungi growing on special substrates - Arrhenia lobata on mosses and Crinipellis scabella on grasses.

One patch included in the community with Cirsium arvense and Solidago canadensis is very poor in macromycete species (3). Only Marasmius oreades occurs here frequently and abundantly.

The community with Betula pendula also has poor fungi (Tab. 7.1). Only saprotrophic species which form small sporocarps, such as Panaeolus foemisecii, occur here, infrequently.

The vast majority of macromycetes identified in the abandoned lands are saprotrophic terrestrial and mycorrhizal fungi. There is a small group of species which grow on grasses, mosses, pine needles and cones. Only two species of lignicolous fungi were observed in several study plots: Schizophyllum commune and Trichaptum abietinum, which grew on branches or twigs of Pinus sylvestris (Tab. 7.1). 

Auriscalpium vulgare Gray

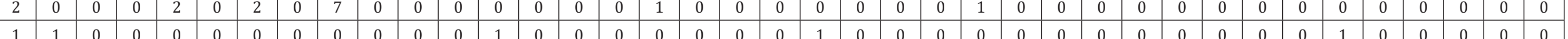
Boletus edulis Bull.: Fr. Bovista nigrescens Pers.: Pers. Bovista plumbea Pers.: Pers.

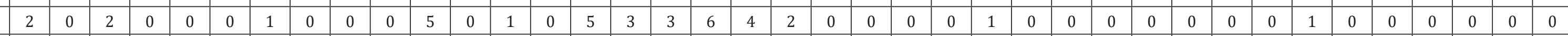

Calocybe gambosa Fr. Donk

Calvatia excipuliformis (Scop.: Pers.) Perdeck

Calvatia utriformis (Bull.: Pers.) Jaap

Chalciporus piperatus (Bull.: Fr.) Bat.

Clitocybe vibecina (Fr). Quél.

Clitopilus prunulus (Scop.: Fr.) P. Kumm

Conocybe rickeaniana Singer ex P.D. Orton

Conocybe tenera (Schaeff.: Fr.) Fayod

\begin{tabular}{|l|l|l|l|l|l|l|l|l|l|l|l|l|l|l|l|l|l|l|l|l|l|l|l|l|l|l|l|l|l|l|l|l|l|l|l|l|l|l|}
2 & 3 & 3 & 2 & 0 & 0 & 0 & 0 & 1 & 1 & 0 & 0 & 1 & 1 & 1 & 1 & 1 & 0 & 1 & 5 & 1 & 0 & 0 & 1 & 0 & 0 & 0 & 0 & 0 & 0 & 0 & 0 & 1 & 1 & 0 & 0 & 0 & 0 & 0 \\
\hline
\end{tabular} \begin{tabular}{|l|l|l|l|l|l|l|l|l|l|l|l|l|l|l|l|l|l|l|l|l|l|l|l|l|l|l|l|l|l|l|l|l|l|l|l|l|l|l|}
5 & 3 & 0 & 3 & 0 & 0 & 2 & 4 & 3 & 4 & 0 & 2 & 4 & 1 & 5 & 4 & 5 & 5 & 5 & 5 & 8 & 3 & 3 & 40 & 1 & 0 & 0 & 0 & 0 & 0 & 0 & 6 & 5 & 5 & 0 & 0 & 0 & 0 & 0 \\
\hline
\end{tabular} \begin{tabular}{l|l|l|l|l|l|l|l|l|l|l|l|l|l|l|l|l|l|l|l|l|l|l|l|l|l|l|l|l|l|l|l|l|l|l|l|l|l|l|l}
1 & 0 & 2 & 0 & 0 & 0 & 0 & 0 & 2 & 0 & 0 & 0 & 0 & 0 & 0 & 0 & 0 & 0 & 0 & 0 & 0 & 0 & 0 & 0 & 0 & 0 & 0 & 0 & 0 & 0 & 0 & 0 & 0 & 0 & 0 & 0 & 0 & 0 & 0 \\
\hline
\end{tabular}

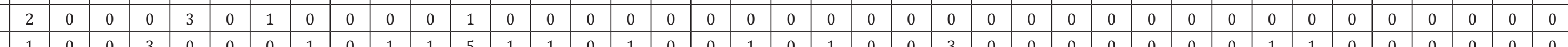

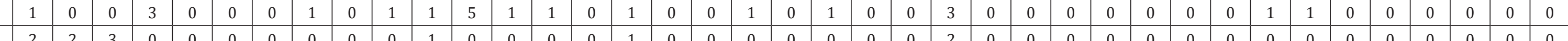

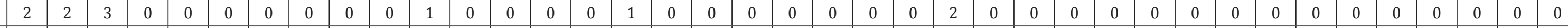

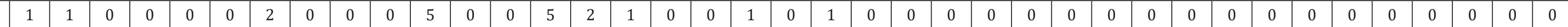
\begin{tabular}{|l|l|l|l|l|l|l|l|l|l|l|l|l|l|l|l|l|l|l|l|l|l|l|l|l|l|l|l|l|l|l|l|l|l|l|l|l|l|l|}
1 & 0 & 0 & 0 & 0 & 0 & 0 & 0 & 0 & 0 & 0 & 0 & 0 & 0 & 1 & 0 & 0 & 0 & 0 & 0 & 0 & 0 & 0 & 0 & 0 & 0 & 0 & 0 & 0 & 0 & 0 & 0 & 0 & 0 & 0 & 0 & 0 & 0 & 0 \\
\hline
\end{tabular} \begin{tabular}{|l|l|l|l|l|l|l|l|l|l|l|l|l|l|l|l|l|l|l|l|l|l|l|l|l|l|l|l|l|l|l|l|l|l|l|l|l|l|l|}
1 & 1 & 0 & 2 & 0 & 0 & 2 & 0 & 2 & 0 & 1 & 0 & 1 & 0 & 1 & 1 & 0 & 0 & 1 & 0 & 0 & 0 & 0 & 0 & 0 & 0 & 0 & 2 & 2 & 0 & 0 & 1 & 1 & 0 & 2 & 0 & 2 & 0 & 1 \\
\hline
\end{tabular}

Crinipellis scabella (Alb. \& Schwein.: Fr.) Murrill \begin{tabular}{llllllllllllllllllllllllllllllll|llllllllllllllll}
6 & 0 & 4 & 0 & 0 & 1 & 1 & 0 & 1 & 1 & 1 & 0 & 1 & 3 & 5 & 1 & 3 & 0 & 1 & 0 & 1 & 0 & 0 & 0 & 2 & 1 & 0 & 0 & 0 & 0 & 0 & 3 & 1 & 5 & 0 & 5 & 0 & 0 & 1 \\
\hline
\end{tabular} Entoloma chalybeum (

Entoloma conferendum (Britzelm.) Noordel. \begin{tabular}{lllllllllllllllllllllllllllllllllllllllllllllllllll}
6 & 0 & 4 & 0 & 0 & 0 & 0 & 0 & 8 & 0 & 0 & 0 & 0 & 0 & 0 & 0 & 0 & 2 & 0 & 0 & 0 & 0 & 0 & 0 & 4 & 0 & 1 & 0 & 0 & 0 & 0 & 0 & 0 & 0 & 0 & 0 & 0 & 0 & 0 \\
\hline 1 & 1 & 0 & 2 & 0 & 1 & 0 & 0 & 0 & 0 & 1 & 2 & 0 & 0 & 0 & 0 & 0 & 0 & 0 & 0 & 0 & 0 & 0 & 0 & 0 & 0 & 0 & 0 & 0 & 0 & 0 & 0 & 0 & 0 & 0 & 0 & 0 & 0 & 0
\end{tabular} Hemimycena lactea (Pers.: Fr.) Singer

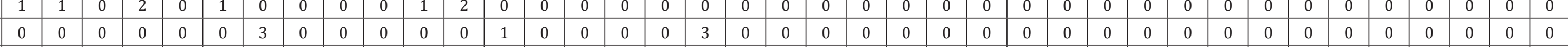

Hygrocybe conica (Schaeff.: Fr.) P. Kumm. \begin{tabular}{l|l|l|l|l|l|l|l|l|l|l|l|l|l|l|l|l|l|l|l|l|l|l|l|l|l|l|l|l|l|l|l|l|l|l|l|l|l|l|}
0 & 0 & 0 & 0 & 0 & 0 & 0 & 0 & 0 & 0 & 0 & 0 & 0 & 0 & 2 & 0 & 0 & 0 & 0 & 0 & 0 & 0 & 0 & 0 & 0 & 0 & 0 & 0 & 0 & 0 & 0 & 0 & 0 & 0 & 0 & 0 & 0 & 0 & 0 \\
\hline
\end{tabular} \begin{tabular}{lllllllllllllllllllllllllllllllllllllllllllllllllllllllllll}
0 & 0 & 0 & 0 & 0 & 0 & 0 & 1 & 0 & 0 & 0 & 0 & 0 & 0 & 0 & 5 & 0 & 0 & 0 & 0 & 0 & 0 & 0 & 0 & 0 & 0 & 0 & 0 & 0 & 0 & 0 & 0 & 1 & 0 & 0 & 0 & 0 & 0 & 0 \\
\hline
\end{tabular} \begin{tabular}{|l|l|l|l|l|l|l|l|l|l|l|l|l|l|l|l|l|l|l|l|l|l|l|l|l|l|l|l|l|l|l|l|l|l|l|l|l|l|l|}
1 & 0 & 0 & 0 & 0 & 1 & 4 & 0 & 0 & 1 & 1 & 0 & 3 & 1 & 1 & 0 & 1 & 1 & 0 & 0 & 1 & 0 & 0 & 0 & 1 & 0 & 1 & 0 & 0 & 0 & 2 & 0 & 1 & 0 & 0 & 1 & 0 & 2 & 0 \\
\hline
\end{tabular} \begin{tabular}{|l|l|l|l|l|l|l|l|l|l|l|l|l|l|l|l|l|l|l|l|l|l|l|l|l|l|l|l|l|l|l|l|l|l|l|l|l|l|l|}
1 & 0 & 0 & 1 & 0 & 0 & 5 & 0 & 0 & 0 & 0 & 0 & 1 & 0 & 0 & 0 & 0 & 0 & 0 & 0 & 0 & 0 & 0 & 0 & 0 & 0 & 1 & 0 & 0 & 0 & 0 & 0 & 0 & 1 & 0 & 0 & 0 & 0 & 0 \\
\hline
\end{tabular}

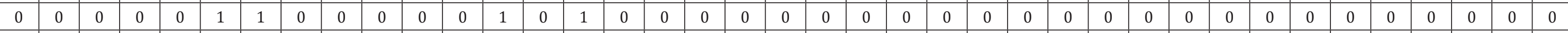
\begin{tabular}{llllllllllllllllllllllllllllllllllllllllllllllllll|l|l|l|l|l|l}
0 & 0 & 0 & 0 & 2 & 0 & 0 & 0 & 0 & 0 & 0 & 0 & 0 & 0 & 0 & 0 & 0 & 0 & 0 & 0 & 0 & 0 & 0 & 0 & 0 & 0 & 0 & 0 & 0 & 0 & 0 & 0 & 0 & 0 & 0 & 0 & 0 & 0 & 0 \\
\hline
\end{tabular} \begin{tabular}{|l|l|l|l|l|l|l|l|l|l|l|l|l|l|l|l|l|l|l|l|l|l|l|l|l|l|l|l|l|l|l|l|l|l|l|l|l|l|l|}
0 & 0 & 0 & 0 & 0 & 5 & 0 & 0 & 0 & 0 & 0 & 0 & 3 & 0 & 0 & 0 & 0 & 0 & 0 & 0 & 0 & 0 & 0 & 0 & 0 & 0 & 2 & 0 & 0 & 0 & 0 & 0 & 0 & 1 & 0 & 0 & 0 & 0 & 0 \\
\hline
\end{tabular}

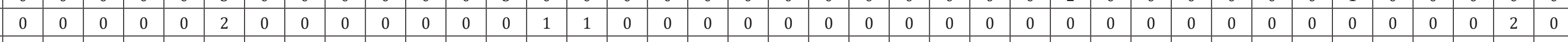

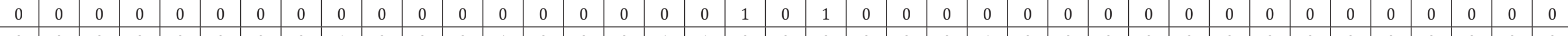

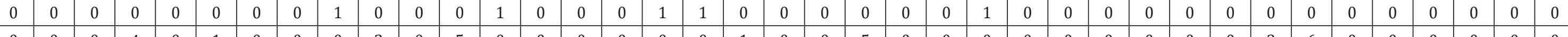
Inocybe maculata Boud. Laccaria laccata (Scop.: Fr.) Berck. \& Broome Leccinum scabrum (Bull.: Fr.) Gray Lepiota alba (Bres.) Sacc. Lycoperdon ericaeum Bonord.

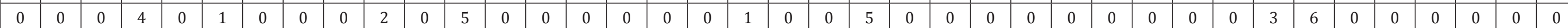

Lycoperdon nigrescens (Pers.: Pers.) Pers. \begin{tabular}{|l|l|l|l|l|l|l|l|l|l|l|l|l|l|l|l|l|l|l|l|l|l|l|l|l|l|l|l|l|l|l|l|l|l|l|l|l|l|l|}
0 & 0 & 0 & 0 & 0 & 2 & 0 & 1 & 0 & 0 & 1 & 0 & 1 & 0 & 1 & 0 & 0 & 5 & 5 & 0 & 1 & 0 & 0 & 0 & 0 & 0 & 0 & 0 & 0 & 0 & 0 & 1 & 1 & 0 & 1 & 2 & 1 & 0 & 0 \\
\hline 5 & 0 & 4 & 0 & 0 & 3 & 0 & 5 & 0 & 3 & 2 & 0 & 5 & 1 & 1 & 1 & 0 & 0 & 0 & 0 & 0 & 0 & 0 & 0 & 3 & 0 & 3 & 3 & 2 & 2 & 5 & 0 & 0 & 0 & 4 & 5 & 2 & 4 & 5 \\
\hline
\end{tabular} Macrolepiota procera (Scop.: Fr.) Singer Marasmius oreades (Bolt.: Fr.) Fr. Mycena epipterygia (Scop.: Fr.) Gray \begin{tabular}{|l|l|l|l|l|l|l|l|l|l|l|l|l|l|l|l|l|l|l|l|l|l|l|l|l|l|l|l|l|l|l|l|l|l|l|l|l|l|l|}
5 & 0 & 4 & 0 & 0 & 3 & 0 & 5 & 0 & 3 & 2 & 0 & 5 & 1 & 1 & 1 & 0 & 0 & 0 & 0 & 0 & 0 & 0 & 0 & 3 & 0 & 3 & 3 & 2 & 2 & 5 & 0 & 0 & 0 & 4 & 5 & 2 & 4 & 5 \\
\hline
\end{tabular}

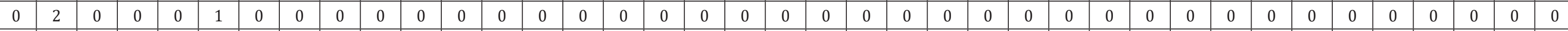

Mycena filopes (Bull.: Fr.) P. Kumm.

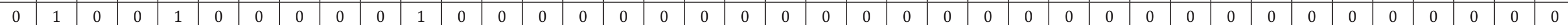

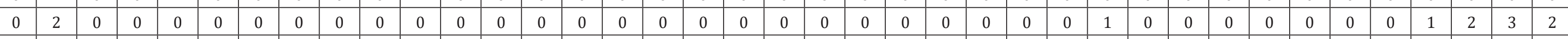
Panaeolus foemisecii (Pers.: Fr.) Kühner Panaeolus fimicola (Fr.) Quél.

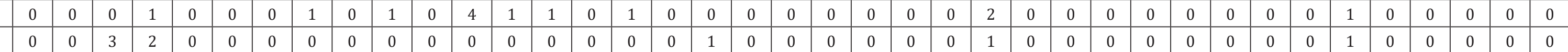
Paxillus involutus (Batsch: Fr.) Fr. ss.lato

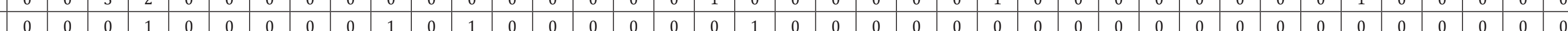
Psilocybe rugosoannulata (Farlow ex Murrill) Noordel. Psilocybe semiglobata (Batsch.: Fr.) Noordel. Psilocybe semilanceata (Fr.) P. Kumm. \begin{tabular}{lllllllllllllllllllllllllllllllllllllll|l|l|l|l|l|l|l|l|l|l|l|l|l|}
0 & 0 & 0 & 0 & 0 & 0 & 0 & 0 & 0 & 0 & 0 & 0 & 0 & 0 & 0 & 0 & 0 & 0 & 0 & 0 & 0 & 0 & 0 & 0 & 0 & 1 & 0 & 0 & 0 & 0 & 0 & 0 & 0 & 0 & 0 & 0 & 0 & 0 & 0 \\
\hline
\end{tabular} Rickenella fibula (Bull,; Fr.) Raith.

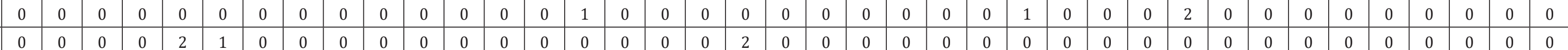


Table 7.1. Frequency of occurrence of macrofungi species in the abandonment study plots in the Łódź Voivodeship (continued)

\begin{tabular}{|c|c|c|c|c|c|c|c|c|c|c|c|c|c|c|c|c|c|c|c|c|c|c|c|c|c|c|c|c|c|c|c|c|c|c|c|c|c|c|c|}
\hline Site/Species & 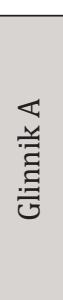 & $\begin{array}{l}\text { 品 } \\
\text { 竘 }\end{array}$ & 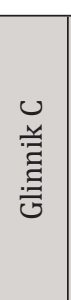 & 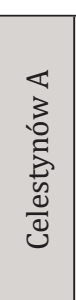 & 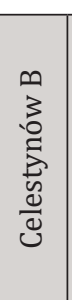 & 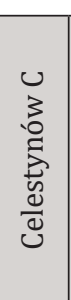 & 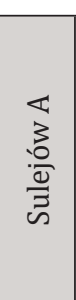 & $\begin{array}{l}\frac{m}{3} \\
\frac{0}{0} \\
\frac{0}{\bar{n}} \\
\bar{n}\end{array}$ & $\begin{array}{l}u \\
\frac{z}{0} \\
\frac{0}{\omega} \\
\bar{\omega}\end{array}$ & 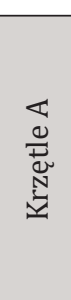 & 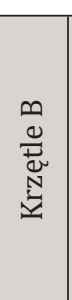 & 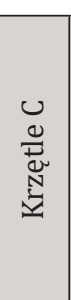 & 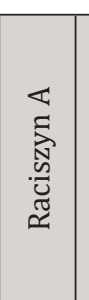 & 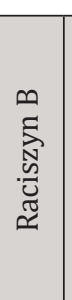 & 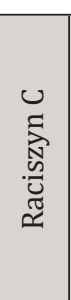 & $\begin{array}{l}\varangle \\
\frac{\pi}{\pi} \\
\frac{\pi}{\pi} \\
\frac{0}{0} \\
3\end{array}$ & 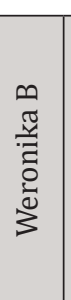 & 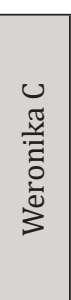 & 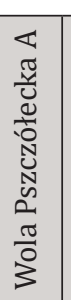 & 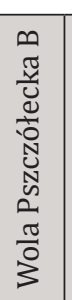 & 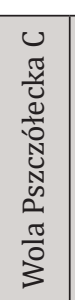 & 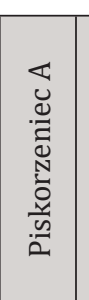 & 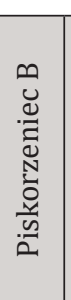 & 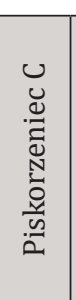 & 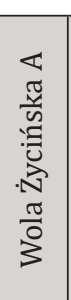 & 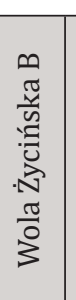 & 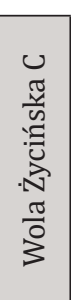 & 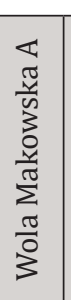 & 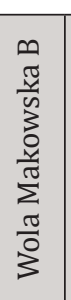 & 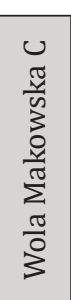 & 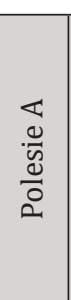 & $\begin{array}{l}\infty \\
\frac{0}{n} \\
\frac{0}{0} \\
0\end{array}$ & 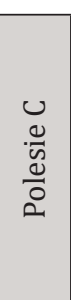 & 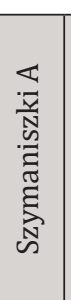 & 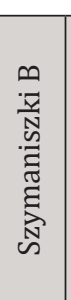 & 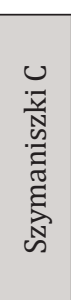 & 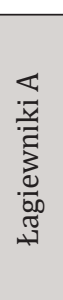 & 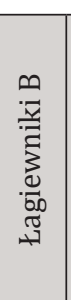 & 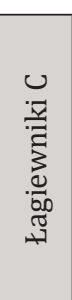 \\
\hline Schizophyllum commune Fr: Fr. & 0 & 0 & 0 & 0 & 0 & 1 & 0 & 0 & 0 & 1 & 0 & 0 & 1 & 1 & 0 & 1 & 4 & 0 & 1 & 0 & 0 & 0 & 0 & 0 & 0 & 0 & 0 & 0 & 0 & 0 & 0 & 1 & 0 & 5 & 0 & 0 & 0 & 0 & 0 \\
\hline Scleroderma citrinum Pers. & 0 & 0 & 0 & 0 & 0 & 1 & 1 & 0 & 0 & 0 & 0 & 0 & 1 & 0 & 0 & 0 & 0 & 0 & 0 & 0 & 0 & 0 & 0 & 0 & 1 & 0 & 2 & 0 & 0 & 0 & 2 & 1 & 0 & 0 & 0 & 0 & 0 & 0 & 0 \\
\hline Suillus bovinus (L.: Fr.) Roussel & 0 & 0 & 0 & 0 & 0 & 2 & 0 & 0 & 0 & 2 & 2 & 0 & 0 & 1 & 1 & 0 & 0 & 0 & 0 & 0 & 0 & 0 & 0 & 0 & 0 & 0 & 0 & 0 & 0 & 0 & 0 & 0 & 0 & 0 & 0 & 0 & 0 & 0 & 0 \\
\hline Suillus luteus (L:: Fr.) Roussel & 0 & 0 & 0 & 0 & 3 & 3 & 0 & 0 & 0 & 0 & 0 & 0 & 0 & 0 & 3 & 0 & 0 & 0 & 0 & 0 & 0 & 0 & 0 & 2 & 0 & 0 & 0 & 0 & 0 & 0 & 0 & 0 & 0 & 0 & 0 & 0 & 0 & 0 & 0 \\
\hline Trichaptum abietinum (Dick:: Fr.) Ryvarden & 0 & 0 & 2 & 0 & 0 & 1 & 0 & 0 & 0 & 0 & 1 & 0 & 0 & 1 & 0 & 0 & 1 & 1 & 0 & 0 & 0 & 0 & 0 & 0 & 0 & 0 & 0 & 0 & 0 & 0 & 0 & 0 & 0 & 0 & 0 & 0 & 0 & 0 & 0 \\
\hline Xerocomus badius (Fr: Fr.) Kühner ex Gilbert & 0 & 0 & 0 & 0 & 0 & 2 & 0 & 0 & 0 & 0 & 0 & 0 & 0 & 1 & 0 & 0 & 0 & 0 & 0 & 0 & 0 & 0 & 0 & 0 & 0 & 0 & 0 & 0 & 0 & 0 & 0 & 0 & 1 & 0 & 0 & 0 & 0 & 0 & 0 \\
\hline
\end{tabular}

Source: own elaboration. 


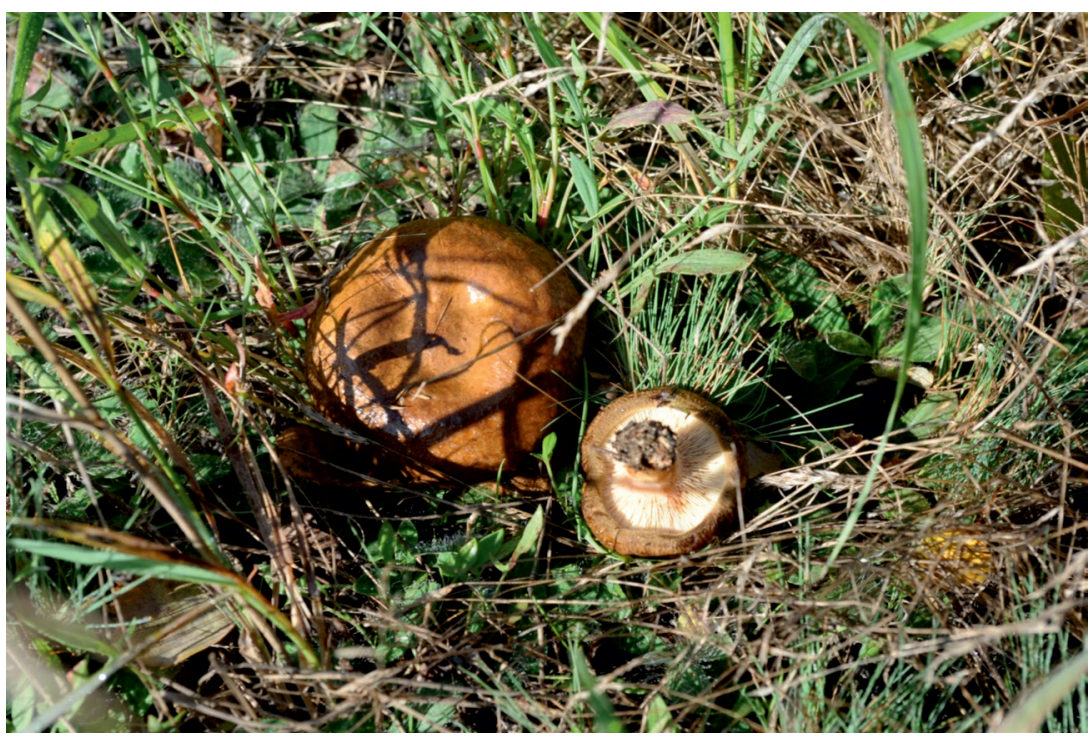

Photo 7.1. Paxillus involutus in Weronika C (E. Papińska)

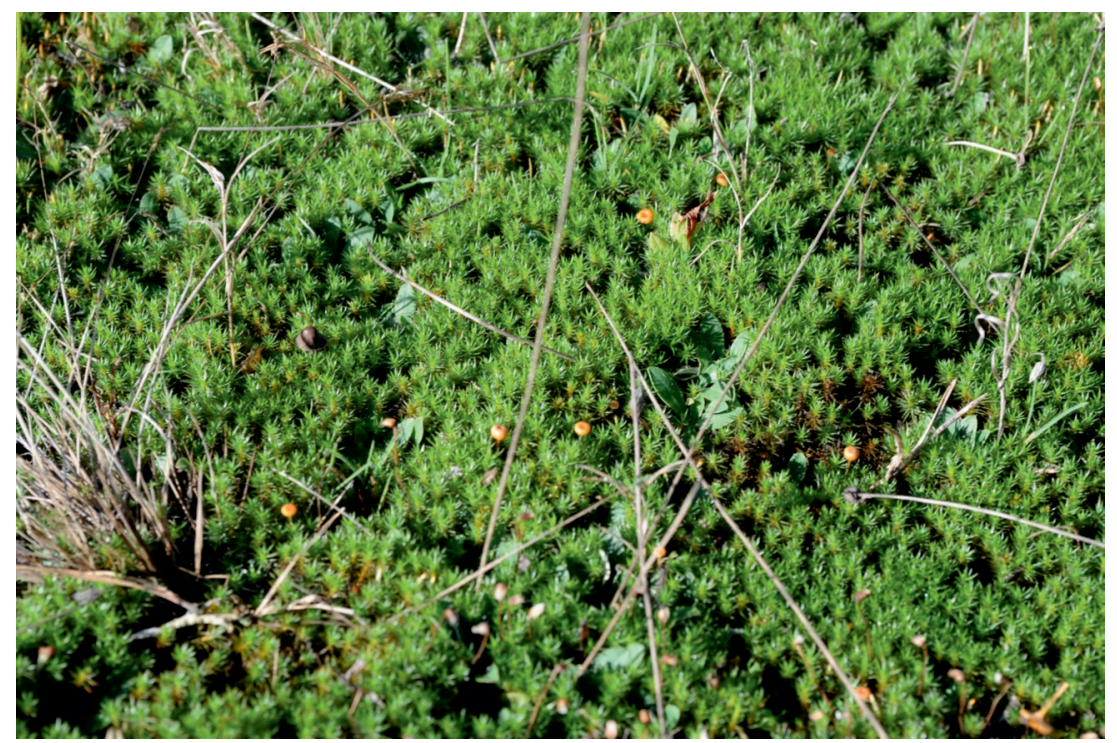

Photo 7.2. Rickenella fibula in Wola Pszczółecka A (E. Papińska)

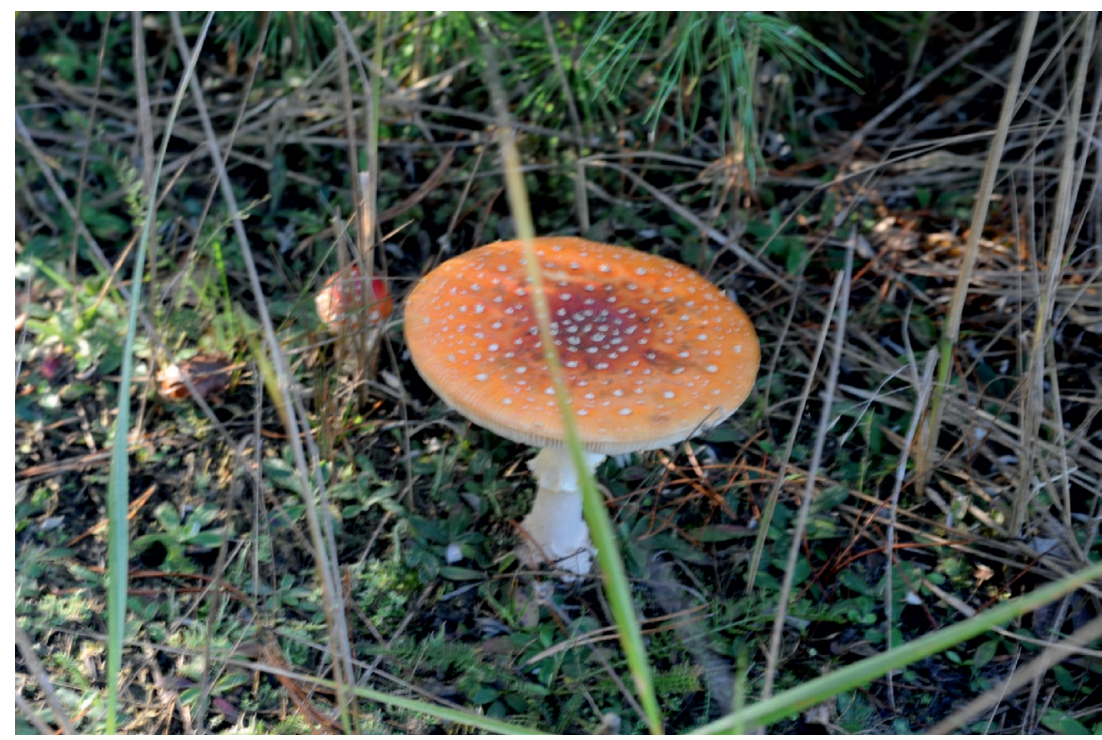

Photo 7.3. Amanita muscaria in Krzętle A (E. Papińska) 


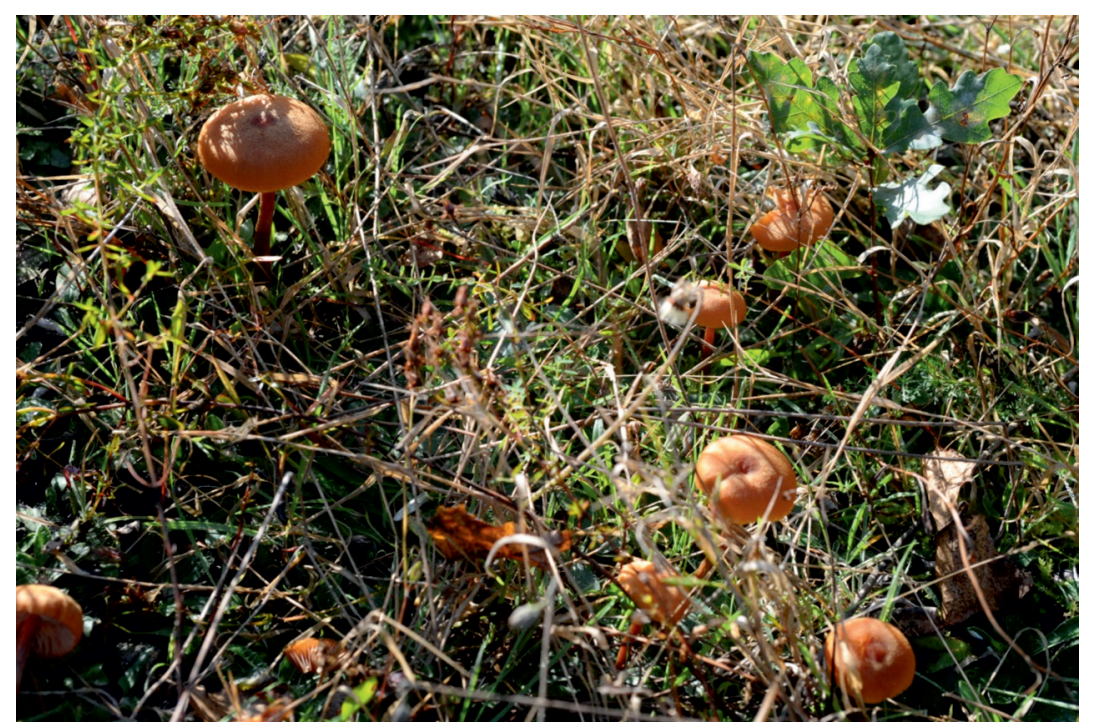

Photo 7.4. Laccaria laccata in Raciszyn A (E. Papińska)

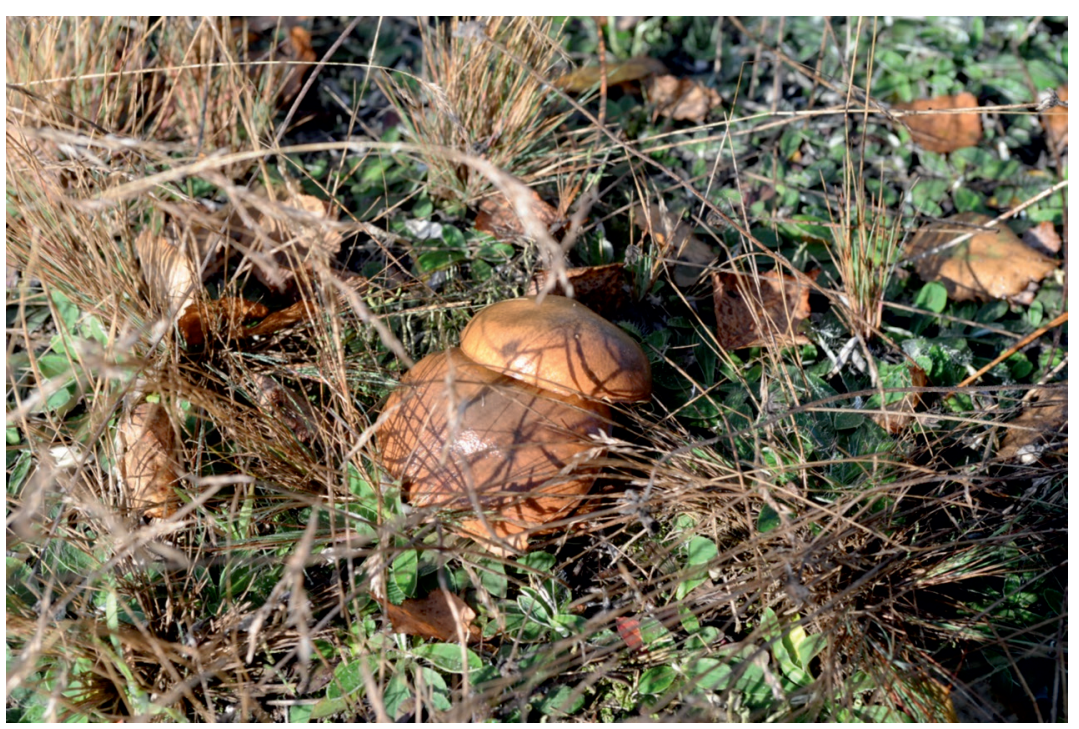

Photo 7.5. Suillus luteus in Raciszyn C (E. Papińska)

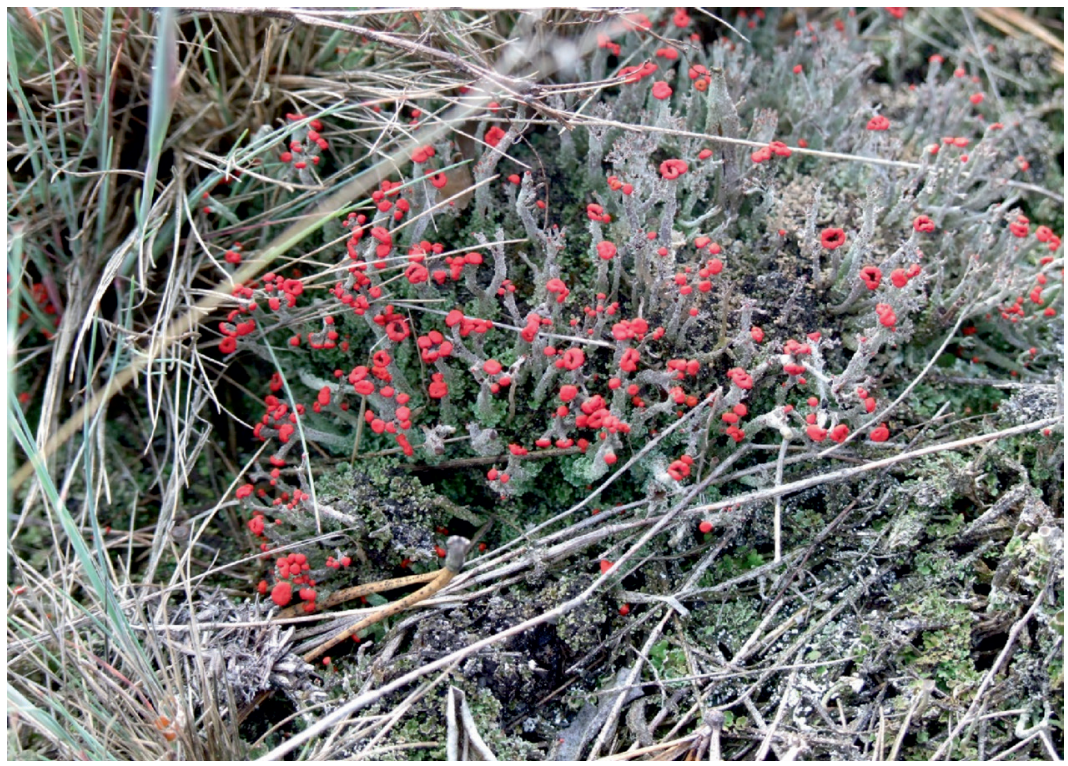

Photo 7.6. Cladonia strepsillis f. coralloides in Polesie A (A. Majchrowska) 